\title{
Implicações da cadência de pedalada sobre a potência mecânica e o período de contração muscular no ciclismo
}

CDD. 20.ed. 616.7

796.6

http://dx.doi.org/10.1590/1807-55092014000300387

\author{
Tiago Canal JACQUES* \\ Fábio Juner LANFERDINI* \\ Rodrigo Rico BINI* \\ Marco Aurélio VAZ*
}

*Escola de Educação

Física, Universidade

Federal do Rio Grande

do Sul.

\section{Resumo}

A energia mecânica necessária para a pedalada no ciclismo depende de ações musculares concêntricas e excêntricas. Contudo, até o momento não se tem conhecimento de como variações na cadência de pedalada podem influenciar o tipo de ação muscular utilizada. 0 presente estudo investigou os efeitos de alterações na cadência nas ações musculares concêntricas e excêntricas durante a pedalada. A absorção e a produção de potência pelas articulações foram calculadas para monitorar possiveis repercussões das mudanças na cadência sobre a cinética articular. Vinte e um ciclistas participaram do estudo $\left(\mathrm{VO}_{2 \text { pico: }}: 64,1 \pm 5.0 \mathrm{ml} / \mathrm{kg} / \mathrm{min}\right.$; volume de treinamento: $368,2 \pm 69,5 \mathrm{~km} /$ semana). Na primeira sessão de avaliação, a potência máxima $\left(\mathrm{PO}_{\mathrm{MAX}}\right)$ e a potência produzida relativa ao segundo limiar ventilatório $\left(\mathrm{PO}_{\mathrm{LV} 2}\right)$ foram determinadas durante teste incremental até a exaustão. Na segunda sessão, os ciclistas realizaram dois testes de dois minutos de duração a 70 e 90 rpm e carga constante $\left(\mathrm{PO}_{\mathrm{LV} 2}\right)$. A ativação de seis músculos, a força aplicada no pedal e a cinemática do membro inferior direito foram avaliadas. Um maior tempo de ativação foi observado em fase excêntrica para os músculos vasto medial $(8 \% ; p<0,01)$ e bíceps femoral $(20 \% ; p=0,04)$ a $70 \mathrm{rpm}$ em relação a $90 \mathrm{rpm}$, além de maior tempo de ativação em fase concêntrica para o músculo vasto medial $(10 \% ; p=0,04)$ a $90 \mathrm{rpm}$ em relação a 70 rpm. Não se observou diferença nas potências articulares entre as cadências testadas. A não alteração da potência articular sugere uma tendência de conservação do padrão do movimento com a alteração da cadência de pedalada. A ativação excêntrica de músculos da articulação do joelho pode estar relacionada com o controle articular, transmissão de força e redução do custo energético.

PalavRas-chave: EMG; Cinética articular; Cinemática articular; Ciclo alongamento-encurtamento.

\section{Introdução}

Contraçóes excêntricas são raramente descritas na análise da pedalada no ciclismo, apesar da indicação de que este tipo de contração estaria associado à transferência de força entre as articulaçóes ${ }^{1-2}$. Contraçôes excêntricas máximas apresentam menor atividade muscular e custo energético quando comparadas a contraçóes concêntricas máximas devido a maior contribuição dos componentes não contráteis em paralelo e em série do musculoesquelético na produção de força ${ }^{2}$.

A energia mecânica requerida para direcionar os pedais durante a pedalada é dependente em grande parte da ação muscular para a produção de potência pelas articulaçóes do quadril e do joelho ${ }^{3}$. Porém, movimentos cíclicos como a pedalada requerem complexas estratégias musculares para permitir a transferência mecânica de energia por meio de músculos bi-articulares ${ }^{4}$. Dentre essas estratégias, foi sugerido que uma porção da energia mecânica aplicada ao pedivela pode estar relacionada ao uso de elementos elásticos não contráteis através do ciclo alongamento-encurtamento, particularmente pela ação dos músculos da articulação do tornozelo5. Porém, até o momento não se tem conhecimento de como variaçốes na cadência de pedalada podem influenciar o tipo de açáo muscular utilizada. A menor atividade muscular para uma determinada carga de trabalho ocorre em uma cadência específica ${ }^{6}$, e, portanto, variaçóes na cadência de pedalada com a manutenção da carga de trabalho podem resultar em 
alteração da mecânica muscular e/ou articular em função de alterações na atividade muscular. Estudos prévios observaram redução no trabalho mecânico do joelho associado à redução na amplitude de movimento das articulaçóes do joelho e do tornozelo em ciclistas pedalando em cadências elevadas (> 90 $\mathrm{rpm})^{7-8}$. No entanto, a duração das contraçốes concêntricas e excêntricas e sua influência no trabalho mecânico articular náo foram quantificadas em diferentes cadências de pedalada. Esta análise permitiria observar se mudanças na duração das contrações concêntricas e excêntricas poderiam explicar possíveis mudanças no trabalho articular. Além disso, o entendimento dos períodos de

\section{Método}

\section{Participantes}

Vinte e um ciclistas, com idade de $28 \pm 6,6$ anos, massa corporal de $70 \pm 6,8 \mathrm{~kg}$, estatura de $177 \pm 9,7$ $\mathrm{cm}$, potência máxima produzida no teste incremental de $375 \pm 30 \mathrm{~W}$, pico do consumo de oxigênio de $64 \pm 5,0 \mathrm{ml} / \mathrm{kg} / \mathrm{min}$, segundo limiar ventilatório de $52 \pm 6,2 \mathrm{ml} / \mathrm{kg} / \mathrm{min}$ e potência produzida no segundo limiar ventilatório de $314 \pm 49,4 \mathrm{~W}$ participaram do estudo. Antes do início das sessôes de avaliação, todos os procedimentos foram informados aos ciclistas, os quais assinaram um termo de consentimento aprovado pelo Comitê de Ética em Pesquisa com Seres Humanos da instituição onde o estudo foi realizado (número 17684).

\section{Primeira sessão}

Na primeira sessão de avaliação, medidas antropométricas (estatura e massa corporal) foram obtidas de acordo com protocolos da Sociedade Internacional para o Avanço da Cineantropometria9. Após, os ciclistas realizaram aquecimento a uma carga de 150 W durante 10 minutos antes do início do teste. Os ciclistas foram avaliados em suas próprias bicicletas acopladas a um ciclo simulador (Computrainer, ProLab 3D, Racermate Inc., Seattle, WA, USA) para a determinação da produção máxima de potência e do pico do consumo de oxigênio. O protocolo consistiu de um período de três minutos de pedalada com carga de trabalho de $100 \mathrm{~W}$ seguido de incrementos de $25 \mathrm{~W}$ a cada minuto. A cadência de pedalada foi visualmente controlada a $90 \pm 2 \mathrm{rpm}$ para todos os ativação concêntrica e excêntrica permite identificar a efetividade da pedalada em cadências elevadas para minimizar a sobrecarga muscular, o que é defendido na prescriçáo do treinamento de ciclistas. Os efeitos destas fases sobre o trabalho mecânico produzido e absorvido pelas articulaçóes atuantes no movimento possibilita sugerir prioridades no treinamento complementar (e.g. treinamento de força) visando fases concêntricas ou excêntricas do gesto motor.

Desta forma, o presente trabalho comparou o período de ativação concêntrica e excêntrica, assim como a potência produzida e absorvida pelas articulaçóes do quadril, joelho e tornozelo, em duas diferentes cadências de pedalada.

ciclistas utilizando-se o "feedback" visual fornecido pelo controlador do ciclo simulador. O consumo de oxigênio foi medido por meio de um sistema de troca gasosa em circuito aberto (CPX/D, Medical Graphics Corp., St Louis, USA). Previamente ao teste incremental, concentraçóes conhecidas de $\mathrm{O}_{2}$ e $\mathrm{CO}_{2}$ foram utilizadas para calibração do sistema. O pico do consumo de oxigênio foi definido quando se observou reduçáo da cadência de pedalada em 10 rpm ou mais, estabilizaçáo do consumo de oxigênio e os valores de quociente respiratório apresentaramse maiores do que 1.1, associadas a possível exaustáo dos ciclistas ${ }^{10}$. O segundo limiar ventilatório foi determinado utilizando a análise dos equivalentes ventilatórios descrita por Weston e GABBETT ${ }^{11}$.

\section{Segunda sessão}

$\mathrm{Na}$ segunda sessão, os ciclistas realizaram aquecimento com carga de $150 \mathrm{~W}$ durante 10 minutos antes do início do teste. Após, pedalaram por um minuto com cadência de $90 \pm 2 \mathrm{rpm}$ e carga relativa a potência máxima produzida durante o teste incremental $\left(\mathrm{PO}_{\mathrm{Max}}\right)$. Após dois minutos de repouso sobre a bicicleta, os ciclistas realizaram dois testes de dois minutos de duração com a potência produzida relativa ao segundo limiar ventilatório $\left(\mathrm{PO}_{\mathrm{LV} 2}\right)$ e cadência controlada em $70 \pm 2 \mathrm{rpm}$ e $90 \pm 2 \mathrm{rpm}$, com dois minutos de intervalo entre os testes. A ordem de execução de cada cadência foi sorteada entre os ciclistas.

Durante as duas avaliaçôes os ciclistas pedalaram em suas próprias bicicletas acopladas ao ciclo simulador. Avaliações da ativação muscular, da força 
aplicada no pedal e da cinemática foram realizadas para o membro inferior direito, sendo os dados obtidos durante os últimos 20 segundos de cada teste.

\section{Coleta dos dados}

Os componentes normal e ântero-posterior da força aplicada ao pedal direito foram mensurados utilizando um pedal dinamométrico $2 \mathrm{D}^{12}$. Os dados de força foram amplificados (ENTRAN MSC6, StrainSense Limited, Northants, UK) e gravados com uma frequência de amostragem de $600 \mathrm{~Hz}$ por canal com a utilização de um conversor de sinais analógico-digital de 16-bits de resolução (DI220, Dataq Instruments, USA) por meio de "software" específico (WINDAQ, DataQ Instruments Inc., USA). A cinemática do membro inferior direito foi monitorada utilizando como pontos de referência para os eixos de rotação das articulaçóes do quadril, joelho e tornozelo marcadores reflexivos posicionados sobre a espinha ilíaca ântero-superior, o trocânter maior do fêmur, o côndilo lateral do fêmur, o maléolo lateral e os limites anterior e posterior do pedal. Dois marcadores foram aderidos ao quadro da bicicleta a uma distância conhecida para calibraçáo das coordenadas espaciais. Uma câmera posicionada perpendicularmente ao plano de movimento (AVT PIKE F-032, Allied Vision Technologies GmbH, Germany) foi utilizada para gravar vídeos a $60 \mathrm{~Hz}$ utilizando o "software" específico (AVT ActiveCam, Allied Vision Technologies GmbH, Germany). As atividades musculares foram obtidas com o uso da eletromiografia de superfície para os músculos vasto medial (VM), reto femoral (RF), bíceps femoral $(\mathrm{BF})$, tibial anterior (TA), gastrocnêmio medial (GM) e sóleo (SOL) utilizando um eletromiógrafo de oito canais (Octopus AMT-8, Bortec Electronics Inc., Canada), com modo de rejeição comum de ruídos de $115 \mathrm{~dB}$ a $60 \mathrm{~Hz}$. Pares de eletrodos Ag/ $\mathrm{AgCl}$ (configuração bipolar) com um diâmetro de $22 \mathrm{~mm}$ foram posicionados na pele após depilação do local e limpeza da área utilizando-se algodão embebido em álcool (70\%) para reduzir a impedância da pele como recomendado pela Sociedade Internacional de Eletrofisiologia e Cinesiologia ${ }^{13-14}$. Os eletrodos foram posicionados sobre a pele na região do ventre muscular, a um terço do comprimento muscular a partir do ponto central (para evitar a junção miotendínea), paralelamente ao direcionamento estimado das fibras musculares. Um eletrodo de referência foi posicionado sobre uma proeminência óssea eletricamente neutra (face anterior da tíbia). Os fios dos eletrodos foram fixados à pele para reduzir ruídos oriundos do movimento de cabos sobre o sinal de eletromiografia. Os dados de eletromiografia de superfície foram gravados (sem uso de filtro analógico) com $1 \mathrm{~K}$ de ganho e $2100 \mathrm{~Hz}$ por canal empregando um conversor de sinal-digital de 16-bits de resolução (DI720, Dataq Instruments, USA) por meio de software específico (WINDAQ, DataQ Instruments Inc., USA). Todos os sistemas foram sincronizados utilizando-se uma unidade de sincronização de eventos desenvolvida de forma customizada de forma a produzir um sinal analógico para os conversores analógico-digitais simultaneamente a um indicador visual para a câmera ${ }^{15}$.

\section{Análise dos dados}

Os arquivos de vídeo contendo o movimento dos ciclistas foram analisados para recorte dos períodos de interesse posteriores ao evento de sincronização utilizando "software" específico (Virtual Dub). Os marcadores reflexivos foram então rastreados automaticamente utilizando software para a análise de movimento (Skill Spector 1.3.2, Video4Coach, Denmark) com o objetivo de obter as coordenadas espaciais bidimensionais $(x-y)$ ao longo do tempo. As coordenadas cinemáticas foram suavizadas por meio de um filtro tipo passa-baixa Butterworth de dupla entrada de segunda ordem com frequência de corte inicial de $5 \mathrm{~Hz}$ e otimização da frequência de corte para a redução dos resíduos do sinal ${ }^{16}$. Os ângulos das articulações do quadril, joelho e tornozelo durante a pedalada foram calculados de forma que os comprimentos das unidades músculo-tendão (VM, RF, BF, TA, GM e SOL) fossem estimados utilizando o modelo de Hawkins e Hull ${ }^{17}$ por meio de rotina customizada desenvolvida em MATLAB $^{\circ}$ (Mathworks Inc, Natick, MA, USA). Para a análise dos sinais de eletromiografia, os sinais foram filtrados usando filtro tipo passa-banda Butterworth de dupla entrada e quinta ordem com frequências de corte iniciais de $20-500 \mathrm{~Hz}$, aprimoradas para redução dos resíduos de sinal ${ }^{16}$. O envelope RMS foi calculado para cada sinal utilizando janelamento retangular com dimensôes de $40 \mathrm{~ms}^{18}$. O RMS de cada músculo foi recortado para a obtenção da média de 10 revoluçôes consecutivas do pedivela para cada músculo de cada ciclista. Os envelopes RMS foram então normalizados pelo valor médio do RMS obtido no teste com carga relativa à $\mathrm{PO}_{\mathrm{MAX}}$. A análise dos dados de eletromiografia foi realizada 
utilizando-se uma rotina customizada desenvolvida em MATLAB ${ }^{\oplus}$ (Mathworks Inc, Natick, MA, USA).

$\mathrm{O}$ início e o final da ativação muscular foram determinados utilizando-se um limiar de 10\% da ativação máxima muscular ${ }^{19}$. Contrações concêntricas foram definidas quando se observou de forma simultânea um encurtamento da unidade músculotendão e o início da ativação muscular, sendo o alongamento da unidade músculo-tendão associado à ativação muscular utilizada para a definição da contração excêntrica ${ }^{20}$.

Após a filtragem dos sinais de força (filtro passa-baixa Butterworth de dupla entrada com frequência de corte inicial de $10 \mathrm{~Hz}$ e otimização para a redução dos resíduos do sinal), a dinâmica inversa do membro inferior foi calculada como descrito anteriormente ${ }^{21}$. Com os momentos das articulaçóes do quadril, joelho e tornozelo encontrados, a potência articular foi calculada como o produto do momento articular em Newton.metro pela velocidade angular da articulaçáo em radianos/ segundo. A produção e absorção de potência na articulação foram então definidas como a potência positiva e negativa para cada articulação (FIGURA 1). O percentual do ciclo do pedivela coberto pela produção ou absorção de potência foi computado utilizando-se rotinas customizadas escritas em MATLAB $^{\circledast}$ (Mathworks Inc, Natick, MA, USA).

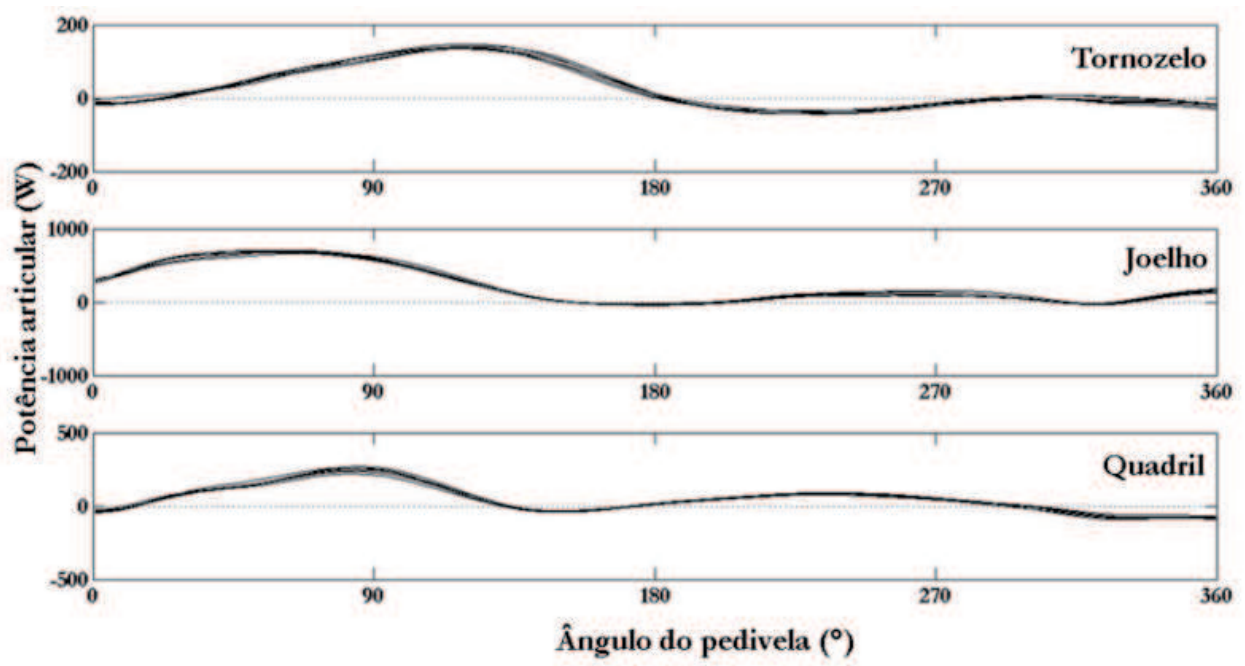

FIGURA 1 - Comportamento da potência articular do tornozelo, joelho e quadril de um ciclista pedalando em carga constante $\left(\mathrm{PO}_{\mathrm{Max}}\right)$.

\section{Análise estatística}

Os dados são apresentados por meio de médias e desvios padrão (DP). A normalidade dos dados foi verificada por meio do teste de Shapiro-Wilk. O teste t para amostras dependentes foi utilizado para comparar os efeitos das cadências (70 e $90 \mathrm{rpm}$ ) na potência articular (quadril, joelho e tornozelo) e no período de ativação concêntrico e excêntrico dos músculos VM, RF, BF, TA, GM e SOL. O "software" SPSS 17.0 foi utilizado para todas as análises, com nível de significância de $\alpha=0,05$.

\section{Resultados}

A duração dos períodos de produção e absorção de potência pelas articulaçóes do quadril, joelho e tornozelo não apresentou diferença significativa entre as cadências testadas (FIGURA 2).

O músculo VM apresentou maior tempo em contração excêntrica na cadência de 70 rpm em comparação a cadência de 90 rpm (8\%; p < 0,01; FIGURA 3), assim como o BF (20\%; p = 0,04; FIGURA 3). O VM apresentou maior tempo em contração concêntrica a 90 rpm em comparação com a cadência de $70 \mathrm{rpm}$ (10\%; $\mathrm{p}=0,04$; FIGURA 3). * diferença significativa entre as cadências para o mesmo tipo de contração $(p<0,05)$. 


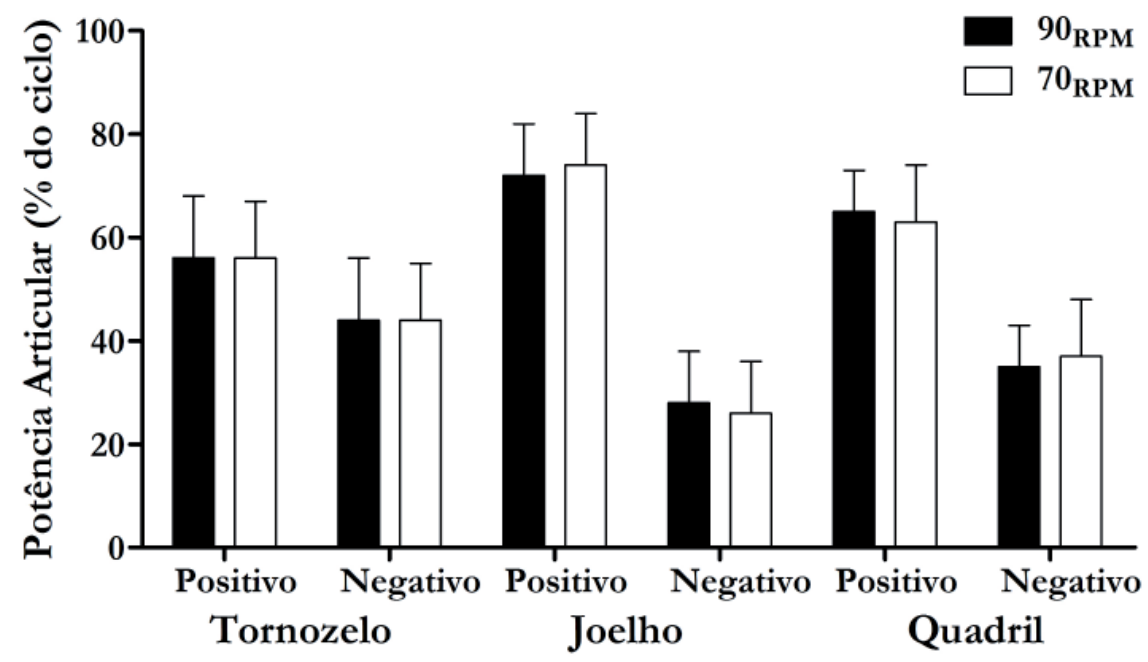

diferença significativa entre as cadências ( $p$ $<0,05)$.

FIGURA 2 - Duração da produção e absorção de potência nas articulações do tornozelo, joelho e quadril nas cadências de 70 e 90 rpm ao longo do ciclo da pedalada.
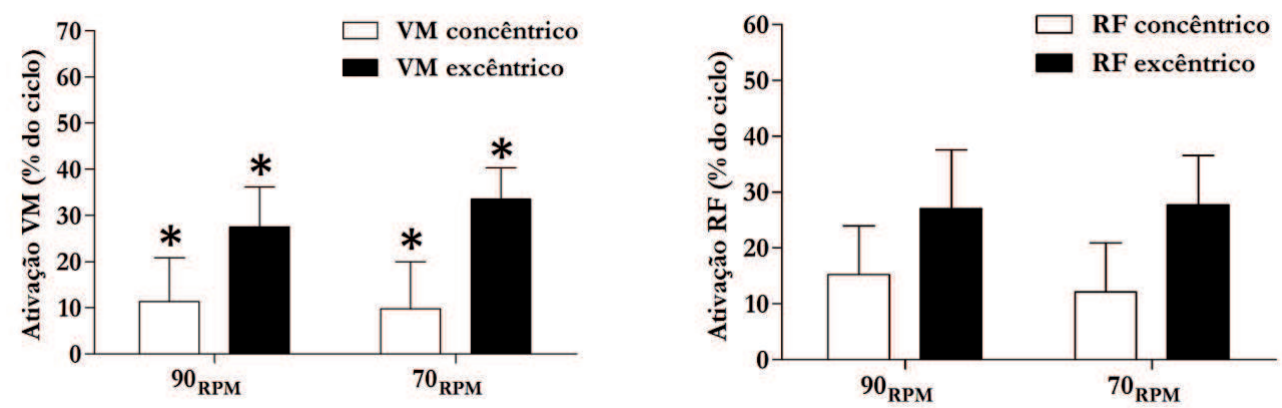

* diferença significativa entre as cadências ( $p$ $<0,05)$.
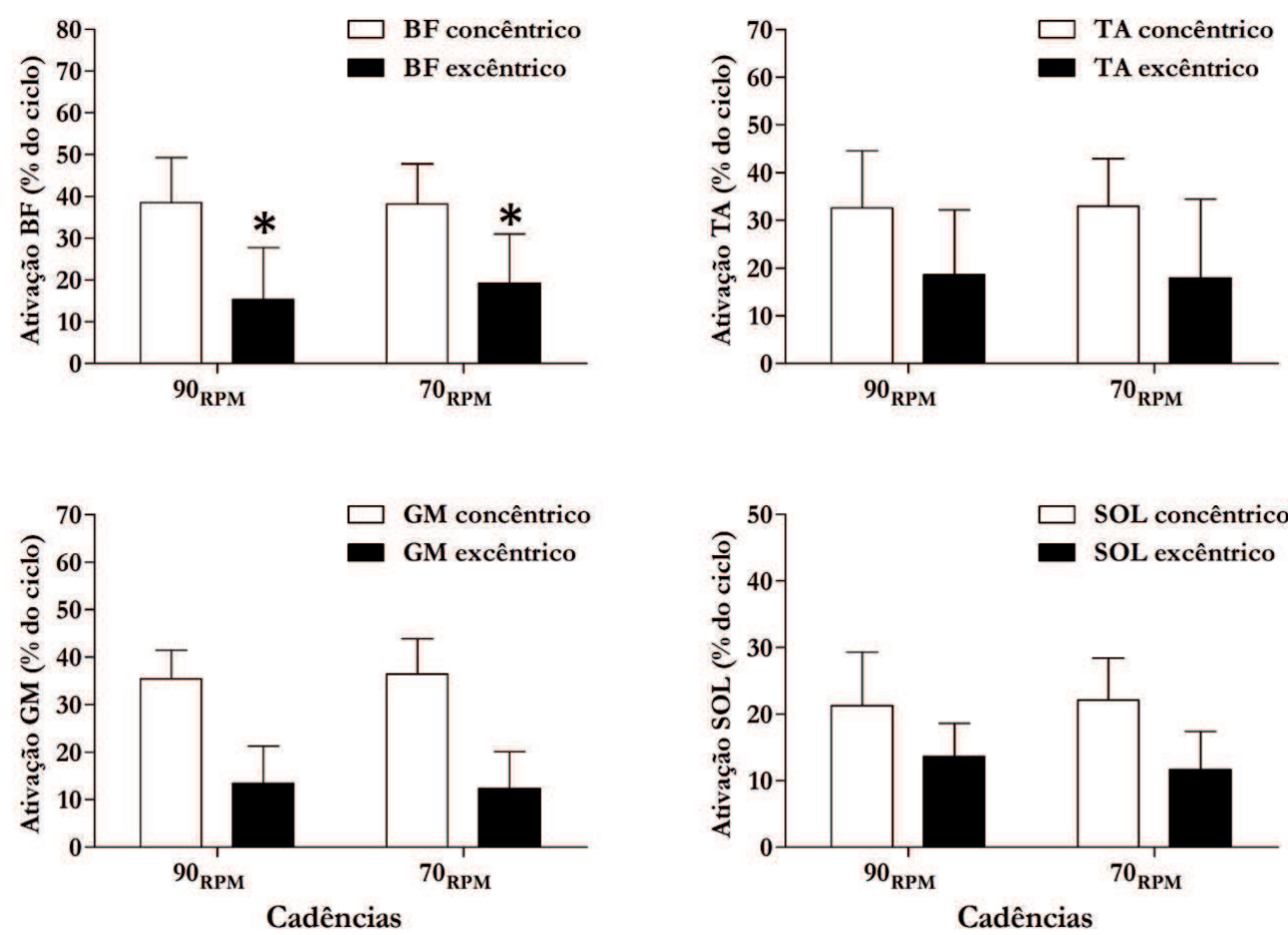

FIGURA 3 - Duração das contrações concêntrica e excêntrica para os músculos vasto medial (VM), reto femoral (RF), bíceps femoral (BF), tibial anterior (TA), gastrocnêmico medial (GM) e sóleo (SOL) para as cadências de 70 e $90 \mathrm{rpm}$ ao longo do ciclo de pedalada. 


\section{Discussão}

Estudos anteriores ${ }^{22-24}$ apontam as relaçóes entre cadência de pedalada e custo energético. No entanto, não há evidências indicando porque cadências menores são mais eficientes. Com isto, objetivamos avaliar se haveriam músculos atuando por mais tempo em ações excêntricas nestas cadências (e.g. $70 \mathrm{rpm}$ ), o que foi observado no presente estudo. Uma vez que nosso estudo é pioneiro na avaliação mútua dos períodos de ativação concêntrica e excêntrica associados ao trabalho mecânico produzido e absorvido pelas articulações, torna-se demasiadamente audacioso determinar fatores definitivos para os fenômenos avaliados. Independentemente, indicamos que as pequenas alteraçóes observadas estejam relacionadas a um consistente programa motor minimamente alterado na ocorrência de mudanças na cadência de pedalada.

Os principais achados do presente estudo estáo relacionados com a identificação de um maior tempo de ativação em contração excêntrica dos músculos VM e BF na cadência de 70 rpm em relação a 90 rpm. Além disso, se observou um maior tempo de ativação em contração concêntrica para o músculo VM na cadência de $90 \mathrm{rpm}$. Não houve diferença na produção e absorção de potência nas articulações do quadril, joelho ou tornozelo entre as cadências testadas. A ausência de alteração na potência articular com a mudança na cadência já foi descrita em estudos prévios ${ }^{7,25-26}$. Os autores sugerem que esta é uma estratégia do sistema nervoso central para reorganizar o recrutamento muscular em função da demanda (cadência e carga de trabalho), regulando o movimento de maneira que a potência articular náo seja afetada e, portanto, preservando o padrão de movimento e o trabalho mecânico articular. Uma vez que no presente estudo a resistência mecânica foi maior a $70 \mathrm{rpm}$, as mudanças na demanda por força muscular nessa cadência podem ter resultado nas alteraçôes observadas no período de ativação das contraçóes concêntricas e excêntricas.

A fase de propulsão $\left(0-180^{\circ}\right)$ é responsável por $-96 \%$ do trabalho mecânico total durante o ciclo da pedalada ${ }^{27}$. Em baixas cadências, há uma redução da tendência de movimento do pedivela em função de propriedades inerciais dos segmentos ${ }^{28}$. É provável que tenha ocorrido uma demanda aumentada por aplicação de força no pedal a $70 \mathrm{rpm}$ em função da menor velocidade dos segmentos nessa cadência. Ainda, sabe-se que contraçóes excêntricas reduzem o custo energético do movimento devido a maior contribuiçáo dos elementos elásticos do músculo esquelético ${ }^{2}$. Dessa forma, o maior tempo de ativação em contração excêntrica do vasto medial e do bíceps femoral a $70 \mathrm{rpm}$ pode ter sido utilizada como estratégia pelo sistema nervoso central para suprir a demanda de força resistiva nas fases de recuperação e propulsão da pedalada, visando a economia de substrato energético. Ainda, a potência produzida no quadril é transmitida pela articulação do joelho durante o ciclo da pedalada, e contraçôes excêntricas são relacionadas à transferência de força entre as articulaçóes ${ }^{1-2}$. Dessa forma, o aumento do período de ativação em contração excêntrica do bíceps femoral e do vasto medial também pode estar ligado a maior necessidade de transferência de força pela articulação do joelho na cadência de 70 rpm em função do papel crucial desta articulação na transmissão de força entre os segmentos do membro inferior.

Em estudos anteriores foi relatado que em maiores velocidades do pedivela a necessidade de produção de força muscular reduz para uma mesma carga, já que componentes inerciais aumentam sua contribuição para o movimento ${ }^{28}$. Com o aumento da velocidade do pedivela, a ênfase na produçáo de força acontece no setor de propulsáo do ciclo da pedalada ${ }^{29}$. Nesse sentido, é possível que a náo alteração do trabalho mecânico a $90 \mathrm{rpm}$ em relação a cadência de $70 \mathrm{rpm}$ seja explicada por meio da redução no tempo de ativação em contração excêntrica para o VM e para o BF. A vantagem mecânica adicionada pelo aumento da velocidade do pedivela e da contribuiçấo de propriedades inerciais dos segmentos resultou em menor necessidade de transmissão de força entre as articulaçóes e menor custo energético a $90 \mathrm{rpm}$, explicando a redução do tempo de ativação em contração excêntrica para o VM e o BF. Dentre as limitaçóes do presente estudo, é importante apontar a análise exclusiva do tempo de contração sem informação quanto a magnitude da produçáo de força durante cada fase de contração. A estratificação da força muscular para os componentes do quadríceps e dos ísquios-tibiais permitiria uma identificaçáo mais clara da funçâo de cada músculo nas cadências avaliadas.

A alteração da cadência de pedalada em carga de trabalho constante não resultou em diferença significativa na potência articular entre as cadências testadas. Especula-se que o sistema nervoso central altere as estratégias neuromusculares com o intuito de manter o padrão coordenativo da pedalada e o 
trabalho mecânico articular. Um maior tempo em contrações excêntricas foi identificado na menor cadência de pedalada para os músculos VM e BF e um maior tempo em contraçóes concêntricas para o VM na maior cadência de pedalada. Dessa forma, se supóe que em baixas cadências de pedalada contraçôes excêntricas são mais utilizadas em função de suas características de maior produção de força e reduzido custo energético, enquanto em altas cadências contraçôes concêntricas são utilizadas em função do aumento da contribuição de propriedades inerciais do pedivela.

\begin{abstract}
Pedaling cadence effects on mechanical power and muscle contraction timing during cycling

The mechanical energy required to propel the crank may depend on eccentric and concentric muscle actions. However, it is uncertain whether pedaling cadence would elicit changes in concentric and eccentric contributions. Therefore, the purpose of the present study was to investigate the effects of alterations in pedaling cadence on the eccentric and concentric muscle actions. Joint power production and absorption were calculated to assess potential effects from variations in pedaling cadence on joint kinetics. Twenty-one cyclists participated in this study $\left(\mathrm{VO}_{2 \text { pico }}: 64.1 \pm 5.0 \mathrm{ml} / \mathrm{kg} / \mathrm{min}\right.$; training volume: $368.2 \pm 69.5 \mathrm{~km} /$ week). In their first session, maximal power output $\left(\mathrm{PO}_{\mathrm{MAX}}\right)$ and power output related to the second ventilation threshold $\left(\mathrm{PO}_{\mathrm{VT}}\right)$ were determined during an incremental maximal cycling test to exhaustion. In their second session, cyclists performed two 2-min trials with workload from their $\mathrm{PO}_{\mathrm{VT} 2}$ at two different cadences (70 and $90 \mathrm{rpm}$ ). Muscle activation of six muscles, pedal forces and lower limb joint kinematics were evaluated. Longer eccentric contraction at $70 \mathrm{rpm}$ for vastus medialis $(8 \% ; p<0.01)$ and biceps femoris $(20 \% ; p=0.04)$ were observed compared to $90 \mathrm{rpm}$. Longer concentric contraction for vastus medialis muscle $(10 \% ; p=0.04)$ at $90 \mathrm{rpm}$ was observed compared to $70 \mathrm{rpm}$. There were no differences in joint power production and absorption among pedaling cadences. No alterations in joint power could indicate maintenance of movement when pedaling cadence is changed. Eccentric contractions from knee muscles could be related to joint control, force transmission and reduced energy cost.
\end{abstract}

KEY WORDS: EMG; Joint kinetics; Joint kinematics; Stretch-shortening cycle.

\title{
Referências
}

1. Bini RR, Diefenthaeler F. Mechanical work and coordinative pattern of cycling. Int J Fundam Appl Kinesiol. 2009;41:25-39.

2. Enoka RM. Eccentric contractions require unique activation strategies by the nervous system. J Appl Physiol. 1996;81:2339-46.

3. Fregly BJ, Zajac FE. A state-space analysis of mechanical energy generation, absorption, and transfer during pedaling. J Biomech. 1996;29:81-90.

4. Van Ingen Schenau GJ, Boots PJM, De Groot G, Snackers RJ, Van Woensel WWLM. The constrained control of force and position in multi-joint movements. Neuroscience. 1992;46:197-207.

5. Neptune RR, Kautz SA, Zajac FE. Muscle contributions to specific biomechanical functions do not change in forward versus backward pedaling. J Biomech. 2000;33:155-64.

6. MacIntosh BR, Neptune RR, Horton JF. Cadence, power, and muscle activation in cycle ergometry. Med Sci Sports Exerc. 2000;32:1281-7.

7. Bini RR, Rossato M, Diefenthaeler F, Carpes FP, Dos Reis DC, Moro ARP. Pedaling cadence effects on joint mechanical work during cycling. Isokinet Exerc Sci. 2010;18:7-13.

8. Sanderson DJ, Martin PE, Honeyman G, Keefer J. Gastrocnemius and soleus muscle length, velocity, and EMG responses to changes in pedalling cadence. J Electromyogr Kinesiol. 2006;16:642-9. 
9. Marfell-Jones M, Olds T, Stewart A, Carter L. International standards for anthropometric assessment: kineanthropometry. Potchefstroom: ISAK; 2006.

10. Bini RR, Carpes FP, Diefenthaeler F, Mota CB, Guimarães ACS. Physiological and electromyographic responses during 40-km cycling time trial: relationship to muscle coordination and performance. J Sci Med Sport. 2008;11:363-70.

11. Weston SB, Gabbett TJ. Reproducibility of ventilation of thresholds in trained cyclists during ramp cycle exercise. J Sci Med Sport. 2001;4:357-66.

12. Nabinger E, Iturrioz I, Zaro MA. Development of a triaxial force platform for the measurement of force at a bicycle pedal. XX International Symposium of Biomechanics in Sports; 2002; Caceres, ESP. Caceres; 2002.

13. Merletti R, Botter A, Troiano A, Merlo E, Minetto MA. Technology and instrumentation for detection and conditioning of the surface electromyographic signal: state of the art. Clin Biomech. 2009;24:122-34.

14. De Luca CJ. The use of surface electromyography in biomechanics. J Appl Biomech. 1997;13:135-63.

15. Muraoka T, Kawakami Y, Tachi M, Fukunaga T. Muscle fiber and tendon length changes in the human vastus lateralis during slow pedaling. J Appl Physiol. 2001;91:2035-40.

16. Winter DA. Biomechanics and motor control of human movement. 3a ed. New Jersey: John Wiley; 2005.

17. Hawkins D, Hull ML. A method for determining lower extremity muscle-tendon lengths during flexion/extension movements. J Biomech. 1990;23:487-94.

18. Neptune RR, Kautz SA, Hull ML. The effect of pedaling rate on coordination in cycling. J Biomech. 1997;30:1051-8.

19. Baum BS, Li L. Lower extremity muscle activities during cycling are influenced by load and frequency. J Electromyogr Kinesiol. 2003;13:181-90.

20. Connick MJ, Li FX. The impact of altered task mechanics on timing and duration of eccentric bi-articular muscle contractions during cycling. J Electromyogr Kinesiol. 2013;23:223-9.

21. Bini RR, Hume PA. Effects of workload and pedalling cadence on knee forces of cyclists. Sports Biomech. 2013;12:93-107.

22. Candotti CT, Loss JF, Bagatini D, et al. Cocontraction and economy of triathletes and cyclists at different cadences during cycling motion. J Electromyogr Kinesiol. 2009;19:915-21.

23. Marsh AP, Martin PE, Sanderson DJ. Is a joint moment-based cost function associated with preferred cycling cadence? J Biomech. 2000;33:173-80.

24. Takaishi T, Yamamoto T, Ono T, Ito T, Moritani T. Neuromuscular, metabolic, and kinetic adaptations for skilled pedaling performance in cyclists. Med Sci Sports Exerc. 1998;30:442-9.

25. Ettema G, Loras H, Leirdal S. The effects of cycling cadence on the phases of joint power, crank power, force and force effectiveness. J Electromyogr Kinesiol. 2009;19:e94-e101.

26. Mornieux G, Guenette JA, Sheel AW, Sanderson DJ. Influence of cadence, power output and hypoxia on the joint moment distribution during cycling. Eur J Appl Physiol. 2007;102:11-8.

27. Coyle EF, Feltner ME, Kautz SA, et al. Physiological and biomechanical factors associated with elite endurance cycling performance. Med Sci Sports Exerc. 1991;23:93-107.

28. Neptune RR, Herzog W. The association between negative muscle work and pedaling rate. J Biomech. 1999;32:1021-6.

29. Rossato M, Bini RR, Carpes FP, Diefenthaeler F, Moro ARP. Cadence and workload effects on pedaling technique of well-trained cyclists. Int J Sports Med. 2008;29:746-52.

\section{Agradecimentos}

Os autores agradecem o apoio financeiro da CAPES e CNPq.

\begin{tabular}{|c|c|}
\hline $\begin{array}{r}\text { ENDEREÇO } \\
\text { Tiago Canal Jacques } \\
\text { Laboratório de Pesquisa do Exercício } \\
\text { Escola de Educação Física } \\
\text { Universidade Federal do Rio Grande do Sul } \\
\text { R. Felizardo, 750 - Jd. Botânico } \\
\text { 90690-200 - Porto Alegre - RS - BRASIL } \\
\text { e-mail: tiago.jacques@ufrgs.br }\end{array}$ & $\begin{array}{l}\text { Recebido para publicação: 03/09/2013 } \\
\text { Revisado: 05/12/2013 } \\
\text { Aceito: 10/06/2014 }\end{array}$ \\
\hline
\end{tabular}

